\title{
Low SNR Capacity for MIMO Rician and Rayleigh-Product Fading Channels with Single Co-channel Interferer and Noise
}

\author{
Caijun Zhong, Shi Jin, Member, IEEE, Kai-Kit Wong, Senior Member, IEEE, \\ Mohamed-Slim Alouini, Fellow, IEEE, and T. Ratnarajah, Senior Member, IEEE
}

\begin{abstract}
This paper studies the ergodic capacity of multipleinput multiple-output (MIMO) systems with a single co-channel interferer in the low signal-to-noise-ratio (SNR) regime. Two MIMO models namely Rician and Rayleigh-product channels are investigated. Exact analytical expressions for the minimum energy per information bit, $E_{b} / N_{0_{\min }}$, and wideband slope, $S_{0}$, are derived for both channels. Our results show that the minimum energy per information bit is the same for both channels while their wideband slopes differ significantly. Further, the impact of the numbers of transmit and receive antennas, the Rician $K$ factor, the channel mean matrix and the interferenceto-noise-ratio (INR) on the capacity, is addressed. Results indicate that interference degrades the capacity by increasing the required minimum energy per information bit and reducing the wideband slope. Simulation results validate our analytical results.
\end{abstract}

Index Terms-Double scattering, fading channels, MIMO systems, optimum combining, performance analysis.

\section{INTRODUCTION}

$\mathbf{S}$ INCE the pioneer work by Winters [1], Telatar [2] and Foschini et al. [3], multiple-input multiple-output (MIMO) antenna systems have received enormous attention. The use of multiple antennas at both ends of the communication link in MIMO systems offers substantial improvement

Paper approved by Prof. Dennis Goeckel, the Editor for Wireless Communication of the IEEE Communications Society. Manuscript received July 2, 2009; revised January 28, 2010.

This work was supported in part by the Engineering and Physical Science Research Council (EPSRC), under Grant EP/G026092/1. The work of S. Jin was supported by National Natural Science Foundation of China under Grants 60902009 and 60925004, and National Science and Technology Major Project of China under Grants 2009ZX03003-005. The work of K. Wong was supported in part by the EPSRC by grant EP/D058716/1 and EP/E022308/1. The work of M. Alouini was supported in part by Qatar National Research Fund (QNRF). The work of T. Ratnarajah was supported by the Future and Emerging Technologies (FET) Programme within the Seventh Framework Programme for Research of the European Commission under FET-Open grant number CROWN-233843. This work was presented in part at 2009 IEEE 10th Workshop on Signal Processing Advances in Wireless Communication, Perugia, Italy, June, 2009.

C. Zhong and T. Ratnarajah are with ECIT, Queen's University Belfast, Northern Ireland, UK, BT3 9DT (e-mail: \{c.zhong, t.ratnarajah\}@ecit.qub.ac.uk).

S. Jin is with the National Mobile Communications Research Laboratory, Southeast University, Nanjing, 210096, P.R. China (e-mail: jinshi@seu.edu.cn).

$\mathrm{K}$. Wong is with the Department of Electrical and Electronic Engineering, University College London, UK (e-mail: k.wong@ee.ucl.ac.uk).

M.-S. Alouini is with King Abdullah University of Science and Technology (KAUST), Thuwal, Mekkah Province, Saudi Arabia (e-mail: alouini@gmail.com).

Digital Object Identifier 10.1109/TCOMM.2010.080310.090366 in capacity and performance over single-antenna systems. Thus far, the analysis of MIMO systems has been extensively investigated for many different statistical channel models of interest (see e.g., [4-7]). Due to spectrum scarcity, nevertheless, communication systems are anticipated to be corrupted by interference, which has motivated the studies of MIMO with interference, e.g., [8-15].

On the other hand, a wide variety of digital communication systems operate at low power where both spectral efficiency and the energy-per-bit can be very low. Examples include wireless sensor networks where low power and energyefficient devices are preferred, and cellular networks where due to frequency reuse, users often operate at low signal-tonoise ratio (SNR) to avoid causing excessive interference to other cell users. It has been shown in $[16,17]$ that $40 \%$ of the geographical locations experience receiver SNR levels below 0 $\mathrm{dB} .{ }^{1}$ In [18], Verdú proposed that the spectral efficiency in the low SNR (or wideband) regime can be analyzed through two parameters; namely, the minimum $E_{b} / N_{0}$ (with $E_{b}$ denoting the average energy per information bit and $N_{0}$ being the noise spectral density) required for reliable communications, and the wideband slope (denoted by $S_{0}$ ). These low SNR metrics not only provide a useful reference in understanding the achievable rate of a channel at low SNRs, but also offer practical insights into the interaction between various system parameters on the capacity performance. In addition, these metrics can be exploited to obtain engineering guidance on the optimal signalling strategies in the low power regime [18].

The low SNR metrics have subsequently been elaborated in [19-23], where the impacts of Rician $K$ factor, spatial correlation, transmit and receiver channel state information (CSI) were investigated. However, all of these works considered the interference-free scenarios, except [19], where the effect of interference was analyzed. The limitation of [19] is that explicit expressions for these two low SNR metrics were only derived for the interference-limited Rayleigh fading channels.

Although [19] developed many profound results towards understanding of the capacity at low SNRs for interferencelimited MIMO Rayleigh fading channels, the corresponding results for Rician fading channels appear to be limited. Rician fading channel is a more general model which captures

\footnotetext{
${ }^{1}$ Since both networks operate in the low power regime and are generally subjected to interference, the analytical results to be developed may find possible applications in both scenarios.
} 
the scenarios with dominant propagation paths between the transmitter and receiver sides, and embraces Rayleigh fading as a special case. Motivated by this and the importance of understanding the capacity of MIMO channels with interference at low SNRs, in this paper, we first investigate the MIMO Rician fading channels with arbitrary mean matrix and $K$ factor, in which we derive exact expressions for $E_{b} / N_{0_{\min }}$ and $S_{0}$ in the presence of both interference and noise. Based on these results, we shall further study the special cases, namely, the multiple-input single-output (MISO) Rician channels, the rank-one deterministic channels and the MIMO Rayleigh channels, in which simple expressions can be obtained to illustrate the impacts of the number of transmit and receive antennas, the Rician $K$ factor, the channel mean matrix, and the interference-to-noise ratio (INR) on the capacity. Also, asymptotic results in the large-system limit and high INR are developed.

Another major contribution of this paper is that we also provide the low SNR capacity analysis for MIMO Rayleighproduct fading channels [24], which recently have emerged as a unified model to describe the reduced-rank phenomenon and bridge the gap between an independent and identically distributed (i.i.d.) full-rank Rayleigh channel and a degenerate rank-one keyhole channel [25]. The matrix product models have also emerged in MIMO multi-hop relaying systems [26, 27]. Due to its importance, the outage performance of MIMO Rayleigh-product channels with interference has been recently studied in [28]. Nonetheless, the capacity of such channels is not at all understood. In contrast, we have made distinctive contributions of understanding the capacity of these channels in the presence of interference by deriving the exact expressions of $E_{b} / N_{0_{\min }}$ and $S_{0}$ at low SNRs. Our assumption is, however, that the co-channel interferer is equipped with only one transmit antenna and as in $[18,19]$ and many similar endeavors, perfect CSI at the receiver side is also assumed.

The reminder of the paper is structured as follows. Section II describes the models for MIMO Rician and Rayleigh-product channels and gives the capacity definition at low SNRs. In Sections III and IV, we derive the low SNR capacity results for MIMO Rician and Rayleigh-product channels, respectively. Numerical results are provided in Section V, and Section VI concludes the paper.

We adopt the following notation. Vectors are written in lowercase boldface letters and matrices are denoted by uppercase boldface letters. The superscripts $(\cdot)^{T},(\cdot)^{*}$ and $(\cdot)^{\dagger}$ represent the transpose, complex conjugate and conjugate transpose operations, respectively. We also use $\operatorname{det}(\cdot)$ and $\operatorname{tr}\{\cdot\}$ to denote the matrix determinant and trace operations, respectively. $\mathbf{I}$ is used to denote an identity matrix of appropriate dimension. $\mathbb{C}^{m \times n}$ stands for an $m \times n$ complex matrix. $\mathrm{E}\{\cdot\}$ returns the expectation, and $\mathcal{C N}\left(\mu, \sigma^{2}\right)$ is a complex Gaussian distribution with mean $\mu$ and variance $\sigma^{2}$.

\section{SySTEM MOdeL}

Consider a communication link with $N_{t}$ transmit and $N_{r}$ receive antennas, corrupted by interference and additive white Gaussian noise (AWGN). The received signals, $\mathbf{y} \in \mathbb{C}^{N_{r} \times 1}$, can be expressed as

$$
\mathbf{y}=\mathbf{H x}+\mathbf{h} s+\mathbf{n},
$$

where $\mathbf{x} \in \mathbb{C}^{N_{t} \times 1}$ is the transmitted symbol vector satisfying $\mathrm{E}\left\{\|\mathbf{x}\|^{2}\right\}=P, s$ is the interference symbol such that $\mathrm{E}\left\{|s|^{2}\right\}=P_{I}$, and $\mathbf{H} \in \mathbb{C}^{N_{r} \times N_{t}}$ denotes the MIMO channel between the transmitter and receiver. Likewise, $\mathbf{h} \in$ $\mathbb{C}^{N_{r} \times 1}$ denotes the channel vector between the interferer and the desired receiver with its entry being i.i.d. zero-mean unit-variance Gaussian random variables, and $\mathbf{n} \in \mathbb{C}^{N_{r} \times 1}$ is the complex AWGN vector with i.i.d. entries following $\mathcal{C N}\left(0, N_{0}\right)$.

In this paper, we investigate the low SNR capacity properties of two important MIMO channel models, namely: 1) Rician fading and 2) Rayleigh-product fading. They are described as follows:

- MIMO Rician channels-In this case, the channel matrix has the structure [29]

$$
\mathbf{H}=\sqrt{\frac{K}{K+1}} \mathbf{H}_{0}+\sqrt{\frac{1}{K+1}} \mathbf{H}_{w},
$$

where $K$ denotes the Rician $K$-factor, and $\mathbf{H}_{w} \in$ $\mathbb{C}^{N_{r} \times N_{t}}$ is the channel matrix containing i.i.d. zero-mean unit-variance complex Gaussian entries. On the other hand, $\mathbf{H}_{0} \in \mathbb{C}^{N_{r} \times N_{t}}$ denotes the channel mean matrix, which is normalized to satisfy

$$
\operatorname{tr}\left\{\mathbf{H}_{0} \mathbf{H}_{0}^{\dagger}\right\}=N_{r} N_{t} .
$$

- MIMO Rayleigh-product channels-The channel matrix $\mathbf{H}$ can be expressed as [24]

$$
\mathbf{H}=\frac{1}{\sqrt{N_{s}}} \mathbf{H}_{1} \mathbf{H}_{2},
$$

where $\mathbf{H}_{1} \in \mathbb{C}^{N_{r} \times N_{s}}$ and $\mathbf{H}_{2} \in \mathbb{C}^{N_{s} \times N_{t}}$ are statistically independent matrices containing i.i.d. zero-mean unit-variance complex Gaussian entries, with $N_{s}$ being the number of effective scatterers. By varying $N_{s}$, this model can describe various rank-deficient effects of a MIMO channel, e.g., it degenerates to Rayleigh fading if $N_{s} \rightarrow \infty$, and a keyhole channel if $N_{s}=1$.

We assume that CSI is not known at the transmitter side but perfectly known at the receiver. Thus, an equal-power allocation policy is used and the ergodic capacity is then expressed as [19]

$$
C=\mathrm{E}\left\{\log _{2} \operatorname{det}\left(\mathbf{I}+\frac{P}{N_{0} N_{t}} \mathbf{H}^{\dagger}\left(\frac{P_{I}}{N_{0}} \mathbf{h}^{\dagger}+\mathbf{I}\right)^{-1} \mathbf{H}\right)\right\} .
$$

As pointed out in [19], with co-channel interference, it is more suitable to define the SNR as

$$
\rho \triangleq\left(\frac{P}{N_{0}}\right) \frac{\mathrm{E}\left\{\operatorname{tr}\left\{\mathbf{H}^{\dagger}\left(\rho_{I} \mathbf{h} \mathbf{h}^{\dagger}+\mathbf{I}\right)^{-1} \mathbf{H}\right\}\right\}}{N_{t} N_{r}},
$$

where $\rho_{I} \triangleq \frac{P_{I}}{N_{0}}$ is regarded as the INR. Based on the definitions, the ergodic capacity expression in (5) can be rewritten as

$$
C(\rho)=
$$




$$
\mathrm{E}\left\{\log _{2} \operatorname{det}\left(\mathbf{I}+\frac{N_{r} \rho \mathbf{H}^{\dagger}\left(\rho_{I} \mathbf{h h}^{\dagger}+\mathbf{I}\right)^{-1} \mathbf{H}}{\mathrm{E}\left\{\operatorname{tr}\left\{\mathbf{H}^{\dagger}\left(\rho_{I} \mathbf{h h}^{\dagger}+\mathbf{I}\right)^{-1} \mathbf{H}\right\}\right\}}\right)\right\} .
$$

At low SNRs, it has proved useful to investigate the capacity in terms of the normalized transmit energy per information bit, $E_{b} / N_{0}$, rather than the per-symbol SNR, $\rho$. This capacity can be well approximated for low $E_{b} / N_{0}$ levels by the following expression [18]

$$
\mathrm{C}\left(\frac{E_{b}}{N_{0}}\right) \approx S_{0} \log _{2}\left(\frac{\frac{E_{b}}{N_{0}}}{\frac{E_{b}}{N_{0} \min }}\right),
$$

in which $\frac{E_{b}}{N_{0}}$ min denotes the minimum energy per information bit required to convey any positive rate reliably and $S_{0}$ is the wideband slope $[18,19]$. These are the two key parameters that dictate the capacity behavior in the low SNR regime, and can be obtained from $C(\rho)$ via $[19]^{2}$

$$
\begin{aligned}
\frac{E_{b}}{N_{0} \text { min }} & =\frac{N_{t} N_{r}}{\mathrm{E}\left\{\operatorname{tr}\left\{\mathbf{H}^{\dagger}\left(\rho_{I} \mathbf{h h}^{\dagger}+\mathbf{I}\right)^{-1} \mathbf{H}\right\}\right\}} \frac{1}{\dot{C}(0)}, \\
S_{0} & =-\frac{2[\dot{C}(0)]^{2}}{\ddot{C}(0)} \ln 2,
\end{aligned}
$$

where $\dot{C}(\cdot)$ and $\ddot{C}(\cdot)$ are, respectively, the first- and secondorder derivatives taken with respect to $\rho$. Note that $\mathrm{C}\left(\frac{E_{b}}{N_{0}}\right)$ implicity captures the second-order behavior of $C(\rho)$ as $\rho \rightarrow$ 0 . It is worth pointing out that though the low SNR metrics are obtained when $\rho \rightarrow 0$, the capacity approximation by (8) is good for SNRs greater than $\frac{E_{b}}{N_{0}}$ min as will be shown in the numerical results section.

\section{RiCIAN MIMO CHANNELS}

In this section, we derive analytical expressions for $E_{b} / N_{0_{\min }}$ and the wideband slope, $S_{0}$, for MIMO Rician fading channels. The main result is given in the following theorem.

Theorem 1: For MIMO Rician fading channels with a single interferer, we have

$$
\begin{aligned}
{\frac{E_{b}}{N_{0} \text { min }}}=\frac{\ln 2}{N_{r}-1+A_{0}}, \\
S_{0}=\frac{2 N_{t}(K+1)^{2}}{2 K+B_{0}},
\end{aligned}
$$

where $A_{0}=D_{1}\left(N_{r}, \rho_{I}\right)$, and $B_{0}$ is shown on the top of next page, and $D_{1}(m, t)$ and $D_{2}(m, t)$ are defined as

$$
\begin{aligned}
& D_{1}(m, t) \triangleq t^{-m} \Psi\left(m, m, t^{-1}\right), \\
& D_{2}(m, t) \triangleq t^{-m} \Psi\left(m, m-1, t^{-1}\right),
\end{aligned}
$$

where $\Psi(\cdot, \cdot, \cdot)$ is the confluent hypergeometric function defined in [30]. ${ }^{3}$

\section{Proof: See Appendix II.}

\footnotetext{
${ }^{2}$ To facilitate comparison to the interference free results, we adopt a slightly different definition of $\frac{E_{b}}{N_{0}}$ min from that in [19]. Specifically, in [19], $E_{b}$ is normalized by the interference energy plus the noise energy while here $E_{b}$ is normalized by the noise energy only. Therefore, the final result of $\frac{E_{b}}{N_{0}}$ differs by a factor of $\rho_{I}+1$.

${ }^{3}$ It should be noted that the confluent hypergeometric function $\Psi$ can be expressed in terms of standard exponential integral function as shown in the Appendix I. Therefore, its computation is well understood.
}

Theorem 1 is general and valid for mean matrix of arbitrary rank, $\mathbf{H}_{0}$, and any possible $N_{t}, N_{r}, K$ and $\rho_{I}$. From (11), we observe that the Rician factor $K$ and the structure of channel mean $\mathbf{H}_{0}$ (as long as $\operatorname{tr}\left\{\mathbf{H}_{0} \mathbf{H}_{0}^{\dagger}\right\}=N_{t} N_{r}$ ) do not affect $E_{b} / N_{0_{\min }}$, while the values of $N_{r}$ and $\rho_{I}$ have a direct impact. Also in (12), we see that all the parameters will affect the wideband slope $S_{0}$.

Based on (11), we can further investigate the impact of $N_{r}$ and $\rho_{I}$ on $E_{b} / N_{0_{\min }}$ as follows.

Corollary 1: The $E_{b} / N_{0_{\min }}$ is a decreasing function of $N_{r}$ (i.e., when $N_{r}$ increases, $E_{b} / N_{0_{\min }}$ decreases) and is an increasing function of $\rho_{I}$ (i.e., when $\rho_{I}$ increases, $E_{b} / N_{0_{\min }}$ increases). Moreover, the increase in $E_{b} / N_{0_{\min }}$ due to interference is upper bounded by $\frac{\ln 2}{N_{r}\left(N_{r}-1\right)}$ for $N_{r} \geq 2$.

Proof: See Appendix III.

Corollary 2: When $\rho_{I} \rightarrow 0$, Theorem 1 reduces to

$$
\begin{aligned}
{\frac{E_{b}}{N_{0} \text { min }}} & =\frac{\ln 2}{N_{r}}, \\
S_{0} & =\frac{2(K+1)^{2}}{\frac{K^{2} \operatorname{tr}\left\{\left(\mathbf{H}_{0} \mathbf{H}_{0}^{\dagger}\right)^{2}\right\}}{N_{t}^{2} N_{r}^{2}}+(1+2 K) \frac{N_{t}+N_{r}}{N_{t} N_{r}}} .
\end{aligned}
$$

In particular, if the channel mean matrix, $\mathbf{H}_{0}$, is of rank-one, then $S_{0}$ can be reduced to

$$
S_{0}=\frac{2(K+1)^{2}}{K^{2}+(1+2 K) \frac{N_{t}+N_{r}}{N_{t} N_{r}}} .
$$

Proof: The results can be obtained with the help of Lemma 3 in Appendix I, together with the fact that $\operatorname{tr}\left\{\left(\mathbf{H}_{0} \mathbf{H}_{0}^{\dagger}\right)^{2}\right\}=N_{t}^{2} N_{r}^{2}$ when $\mathbf{H}_{0}$ is of rank-one.

Corollary 2 corresponds to the results for MIMO Rician fading channels in an interference-free environment, which generalizes the results in [19] where a rank-one channel mean was considered.

To gain further insight, in the following, we look at three special cases: 1) MISO Rician fading channels, i.e., $N_{r}=1$, 2) MIMO Rician channels of rank-one mean for large $K$, i.e., $K \rightarrow \infty$ and $\mathbf{H}_{0}=\boldsymbol{\alpha} \boldsymbol{\beta}^{\dagger}$ (with complex column vectors $\boldsymbol{\alpha}, \boldsymbol{\beta}$ ), and 3) MIMO Rayleigh channels, i.e., $K=0$.

\section{A. MISO Rician Channels}

Corollary 3: For MISO Rician channels, i.e., $N_{r}=1$, we have

$$
\begin{aligned}
\frac{E_{b}}{N_{0 \text { min }}} & =\frac{\ln 2}{D_{1}\left(1, \rho_{I}\right)}, \\
S_{0} & =\frac{2 N_{t}(K+1)^{2} D_{1}\left(1, \rho_{I}\right)^{2}}{2 K+\left[1+N_{t}(1+K)^{2}\right] D_{2}\left(1, \rho_{I}\right)} .
\end{aligned}
$$

Proof: The result can be obtained by substituting $N_{r}=1$ in Theorem 1.

Corollary 4: When $N_{r}=1, S_{0}$ is an increasing function of $N_{t}$. When $0 \leq K<1-D_{2}\left(1, \rho_{I}\right), S_{0}$ is a decreasing function of $K$, while for $K \geq 1-D_{2}\left(1, \rho_{I}\right), S_{0}$ is an increasing function of $K$.

Proof: See Appendix IV.

In contrast to the interference-free case, where the increase of Rician factor $K$ always improves the wideband slope $S_{0}$ when $N_{r}=1$, Corollary 4 reveals that the impact of $K$ on 


$$
\begin{aligned}
B_{0}=\frac{1}{\left(N_{r}-1+D_{1}\left(N_{r}, \rho_{I}\right)\right)^{2}} & {\left[\left(\frac{2 K^{2}\left(\operatorname{tr}\left\{\left(\mathbf{H}_{0} \mathbf{H}_{0}^{\dagger}\right)^{2}\right\}-N_{t}^{2} N_{r}\right)}{N_{t}\left(N_{r}+1\right)}+2\left(N_{r}-1\right)\right) D_{1}\left(N_{r}, \rho_{I}\right)\right.} \\
& +\left(1+(1+2 K) N_{t}+\frac{K^{2}\left(\operatorname{tr}\left\{\left(\mathbf{H}_{0} \mathbf{H}_{0}^{\dagger}\right)^{2}\right\}+N_{t}^{2} N_{r}^{2}\right)}{N_{t} N_{r}\left(N_{r}+1\right)}\right) D_{2}\left(N_{r}, \rho_{I}\right) \\
& \left.+\left(N_{r}-1\right)\left(N_{r}-1+(1+2 K) N_{t}+\frac{K^{2}\left(\operatorname{tr}\left\{\left(\mathbf{H}_{0} \mathbf{H}_{0}^{\dagger}\right)^{2}\right\}\left(N_{r}^{2}-N_{r}-1\right)+N_{t}^{2} N_{r}^{2}\right)}{N_{t} N_{r}\left(N_{r}^{2}-1\right)}\right)\right],
\end{aligned}
$$

$S_{0}$ depends on the interference level. Moreover, when $\rho_{I} \rightarrow$ $\infty, S_{0}=0$ which aligns with the observations in [19] for interference-limited Rayleigh fading scenarios. However, the general impact of $\rho_{I}$ on $S_{0}$ is more difficult to characterize, though simulation results indicate that $S_{0}$ decreases when $\rho_{I}$ increases.

\section{B. Rank-1 Mean MIMO Rician Channels for Large $K$}

Corollary 5: In the large $K$ regime, for rank-one mean MIMO Rician channels with a single interferer, it can be derived that

$$
\begin{aligned}
& {\frac{E_{b}}{N_{0} \text { min }}}^{=}=\frac{\ln 2}{N_{r}-1+A_{0}}, \\
& S_{0}=\frac{2\left(1 / N_{r}+1\right)\left(N_{r}-1+D_{1}\left(N_{r}, \rho_{I}\right)\right)^{2}}{N_{r}\left(N_{r}-1\right)+2\left(N_{r}-1\right) D_{1}\left(N_{r}, \rho_{I}\right)+2 D_{2}\left(N_{r}, \rho_{I}\right)},
\end{aligned}
$$

Proof: The desired results can be obtained by taking the limit $K \rightarrow \infty$ in Theorem 1 .

Corollary 5 indicates that in the large $K$ regime, for rank1 mean Rician MIMO fading channels, multiple transmit antennas are irrelevant in terms of $E_{b} / N_{0_{\min }}$ and $S_{0}$. This is actually an intuitive result. The reason is that the large $K$ regime corresponds to the non-fading channel scenarios, and thus, varying the number of transmit antennas for a fixed total transmit power will not increase the receive signal energy and will not contribute to the capacity. In addition, $N_{r}$ affects both $E_{b} / N_{0 \min }$ and $S_{0}$ in contrast to the interference-free case where $N_{r}$ is only relevant in terms of $E_{b} / N_{0 \text { min }}$ [19].

With the help of Lemma 3, we can further obtain the results in various asymptotic regimes:

- When $\rho_{I} \rightarrow 0$, Corollary 5 reduces to

$$
\begin{aligned}
\frac{E_{b}}{N_{0} \min _{n}} & =\frac{\ln 2}{N_{r}}, \\
S_{0} & =2 .
\end{aligned}
$$

The above results correspond to the interference-free scenario, and conforms to those in [19].

- When $N_{r} \rightarrow \infty$, Corollary 5 reduces to

$$
\begin{aligned}
{\frac{E_{b}}{N_{0} \text { min }}} & =\frac{\ln 2}{N_{r}-1}, \\
S_{0} & =2\left(1-\frac{1}{N_{r}^{2}}\right) \approx 2 .
\end{aligned}
$$

Compared with the interference-free case, the above results suggest that interference degrade the capacity by increasing $E_{b} / N_{0_{\min }}$ and decreasing $S_{0}$. For sufficiently large $N_{r}$, the capacity performance with a single interferer (regardless of the interference power level) is similar to that of a channel with one less receive antenna operating in an interference-free environment.

- When $\rho_{I} \rightarrow \infty$ and $N_{r} \geq 2$, Corollary 5 reduces to

$$
\begin{aligned}
{\frac{E_{b}}{N_{0} \text { min }}} & =\frac{\ln 2}{N_{r}-1}, \\
S_{0} & =2\left(1-\frac{1}{N_{r}^{2}}\right) .
\end{aligned}
$$

Intriguingly, these results coincide with the case $N_{r} \rightarrow$ $\infty$. Nevertheless, it is worth mentioning that the situations in application are very different. One is applicable for large $N_{r}$ but arbitrary interference power $\rho_{I}$, while the other is valid for large $\rho_{I}$ but arbitrary $N_{r}$.

\section{MIMO Rayleigh Channels}

Corollary 6: For MIMO Rayleigh channels with a single interferer, we have

$$
\begin{aligned}
{\frac{E_{b}}{N_{0 \text { min }}}}=\frac{\ln 2}{N_{r}-1+A_{0}}, \\
S_{0}=\frac{2 N_{t}}{1+B_{1}},
\end{aligned}
$$

where $B_{1}$ is defined as

$$
B_{1} \triangleq \frac{N_{t}\left(N_{r}-1\right)+\left(N_{t}+1\right) D_{2}\left(N_{r}, \rho_{I}\right)-D_{1}\left(N_{r}, \rho_{I}\right)^{2}}{\left[N_{r}-1+D_{1}\left(N_{r}, \rho_{I}\right)\right]^{2}} \text {. }
$$

Proof: The results follow immediately by substituting $K=0$ into Theorem 1 .

Corollary 6 shows that the number of transmit antennas affects the capacity performance through $S_{0}$. More insights can be gained by looking into the asymptotic regimes as follows.

- When $\rho_{I} \rightarrow 0$, we have

$$
\begin{aligned}
\frac{E_{b}}{N_{0 \text { min }}} & =\frac{\ln 2}{N_{r}}, \\
S_{0} & =\frac{2 N_{t} N_{r}}{N_{t}+N_{r}} .
\end{aligned}
$$

This scenario corresponds to the case for MIMO Rayleigh channels without interference, and the results are consistent with those derived in [19]. 
- When $N_{r} \rightarrow \infty$, we have

$$
\begin{aligned}
\frac{E_{b}}{N_{0 \text { min }}} & =\frac{\ln 2}{N_{r}-1}, \\
S_{0} & =\frac{2 N_{t}\left(N_{r}-1\right)}{N_{t}+N_{r}-1} .
\end{aligned}
$$

- When $\rho_{I} \rightarrow \infty$ and $N_{r} \geq 2$, we have

$$
\begin{aligned}
\frac{E_{b}}{N_{0 \text { min }}} & =\frac{\ln 2}{N_{r}-1}, \\
S_{0} & =\frac{2 N_{t}\left(N_{r}-1\right)}{N_{t}+N_{r}-1} .
\end{aligned}
$$

Similar to the case of rank-one mean MIMO Rician channels with a large $K$, it is observed that the results for $N_{r} \rightarrow \infty$ and $\rho_{I} \rightarrow \infty$ coincide. In addition, by comparing the above results to the interference-free results, we see that in Rayleigh fading, $E_{b} / N_{0_{\min }}$ and $S_{0}$ for a MIMO channel with a single interferer behaves like a channel with one less receive antenna operating in an interference-free environment, which is different from the large $K$ rank-one mean MIMO Rician channel case where $S_{0}$ does not have this interpretation.

\section{MIMO RAYLEIGH-PRODUCT CHANNELS}

In this section, we develop the low SNR capacity results for MIMO Rayleigh-product channels.

Theorem 2: For MIMO Rayleigh-product channels with a single interferer, we have

$$
\begin{aligned}
{\frac{E_{b}}{N_{0} \min }} & =\frac{\ln 2}{N_{r}-1+A_{0}}, \\
S_{0} & =\frac{2 N_{t} N_{s}}{N_{t}+N_{s}+B_{2}},
\end{aligned}
$$

where $A_{0}$ has been defined in Theorem 1 and $B_{2}$ is shown on top of next page.

Proof: See Appendix V.

Theorem 2 shows that the $E_{b} / N_{0_{\min }}$ for MIMO Rayleighproduct channels is the same as that for MIMO Rician fading channels, although the two channels have very different information-carrying capabilities. As such, the results of Corollary 1 also apply for Rayleigh-product channels. Nonetheless, this is not surprising as has already been reported in [18], and this is the consequence of the noise being additive Gaussian. This explains that $E_{b} / N_{0_{\min }}$ is not sufficient to indicate the capacity performance and motivates the need for higher order approximation of the capacity such as the wideband slope, $S_{0}$, which is generally different for different channels. In addition, it is observed that $N_{t}$ and $N_{s}$ affect the capacity performance through the wideband slope $S_{0}$ but not $E_{b} / N_{0_{\min }}$.

Corollary 7: $S_{0}$ is an increasing function of $N_{s}$ and when $N_{s} \rightarrow \infty$, the wideband slope for Rayleigh-product fading with a single interferer becomes the same as that for Rayleigh fading scenarios.

Proof: See Appendix VI.

The above corollary shows that when the number of scatterers increases, the ergodic capacity improves. Moreover, it indicates that the Rayleigh-product channel converges to a Rayleigh fading channel when $N_{s} \rightarrow \infty$. This result is quite intuitive since the large $N_{s}$ corresponds to a rich scattering environment which is the scenario that fits well with the Rayleigh fading model.

The following asymptotic cases are looked at to gain further understanding.

- When $\rho_{I} \rightarrow 0$, we have

$$
\begin{aligned}
\frac{E_{b}}{N_{0} \text { min }} & =\frac{\ln 2}{N_{r}}, \\
S_{0} & =\frac{2 N_{t} N_{s} N_{r}}{N_{t} N_{s}+N_{r} N_{s}+N_{t} N_{r}+1} .
\end{aligned}
$$

This scenario corresponds to the interference-free case for Rayleigh-product channels whose results have been derived in [32]. In addition, when $N_{s}=1$, we further have

$$
S_{0}=\frac{2 N_{t} N_{r}}{\left(N_{t}+1\right)\left(N_{r}+1\right)}
$$

which provides the wideband slope for keyhole channels.

- When $N_{r} \rightarrow \infty$, we have

$$
\begin{aligned}
{\frac{E_{b}}{N_{0} \text { min }}} & =\frac{\ln 2}{N_{r}-1}, \\
S_{0} & =\frac{2 N_{t} N_{s}\left(N_{r}-1\right)}{N_{t} N_{s}+\left(N_{r}-1\right)\left(N_{s}+N_{t}\right)+1} .
\end{aligned}
$$

- When $\rho_{I} \rightarrow \infty$ and $N_{r} \geq 2$, it can be easily shown that $E_{b} / N_{0_{\min }}$ and $S_{0}$ are, respectively, given by (44) and (45). In other words, the results for $N_{r} \rightarrow \infty$ and $\rho \rightarrow \infty$ coincide. Additionally, similar to MIMO Rayleigh channels, the penalty of having an interferer is illustrated through a reduction on the number of effective receive antennas by 1 .

\section{Numerical Results}

In this section, we perform various simulations to further examine the derived analytical expressions. All the MonteCarlo simulation results were obtained by averaging over $10^{5}$ independent channel realizations. For MIMO Rician channels, the mean matrix is generated according to [31]

$$
\mathbf{H}_{0}=\sum_{l=1}^{L} \beta_{l} \boldsymbol{\alpha}\left(\theta_{r, l}\right) \boldsymbol{\alpha}\left(\theta_{t, l}\right)^{T},
$$

where $\beta_{l}$ is the complex amplitude of the lth path, and $\boldsymbol{\alpha}\left(\theta_{t, l}\right)$ and $\boldsymbol{\alpha}\left(\theta_{r, l}\right)$ are the specular array responses corresponding to the $l$ th dominant path at the transmitter and receiver, respectively. The array response is defined as $\left[1, e^{j 2 \pi d \cos (\theta)}, \cdots, e^{j 2 \pi d(N-1) \cos (\theta)}\right]^{T}$ where $d$ is the antenna spacing in wavelengths. In all simulations, we assume that $d=0.5$ at both the transmit and receive sides.

For $3 \times 2$ MIMO Rician channels, the mean matrix is constructed by assuming that there are two dominant paths (i.e., $L=2$ ), with the arriving and departure angles given by $\theta_{r, 1}=\theta_{t, 1}=\frac{\pi}{2}+\frac{\pi}{8}, \theta_{r, 2}=\theta_{t, 2}=\frac{\pi}{2}-\frac{\pi}{8},{ }^{4}$ respectively. The complex coefficient $\beta_{l}$ is chosen such that $\operatorname{tr}\left\{\mathbf{H}_{0} \mathbf{H}_{0}^{\dagger}\right\}=$ $N_{t} N_{r}$. For rank-1 mean Rician fading MIMO channels, we assume $L=1, \beta_{1}=1$ and $\theta_{r, 1}=\theta_{t, 1}=\frac{\pi}{2}$.

\footnotetext{
${ }^{4}$ These angles are randomly chosen for simulation purpose, and our results are applicable to arbitrary angles.
} 


$$
B_{2} \triangleq \frac{\left(N_{r}-1\right)\left(N_{t} N_{s}+1\right)+\left(N_{t}+1\right)\left(N_{s}+1\right) D_{2}\left(N_{r}, \rho_{I}\right)-\left(N_{s}+N_{t}\right) D_{1}\left(N_{r}, \rho_{I}\right)^{2}}{\left[N_{r}-1+D_{1}\left(N_{r}, \rho_{I}\right)\right]^{2}} .
$$

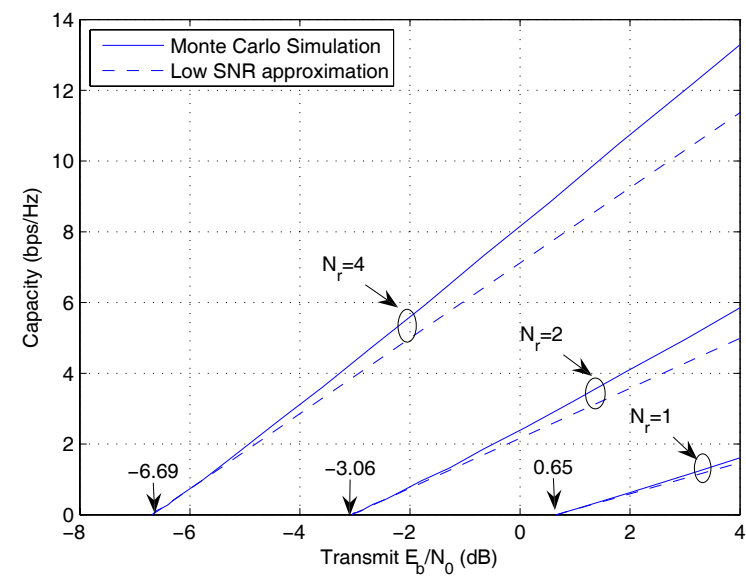

Fig. 1. Low SNR capacity versus transmit $E_{b} / N_{0}$ for Rayleigh fading channels with different $N_{r}$ when $N_{t}=3$ and $\rho_{I}=0 \mathrm{~dB}$.

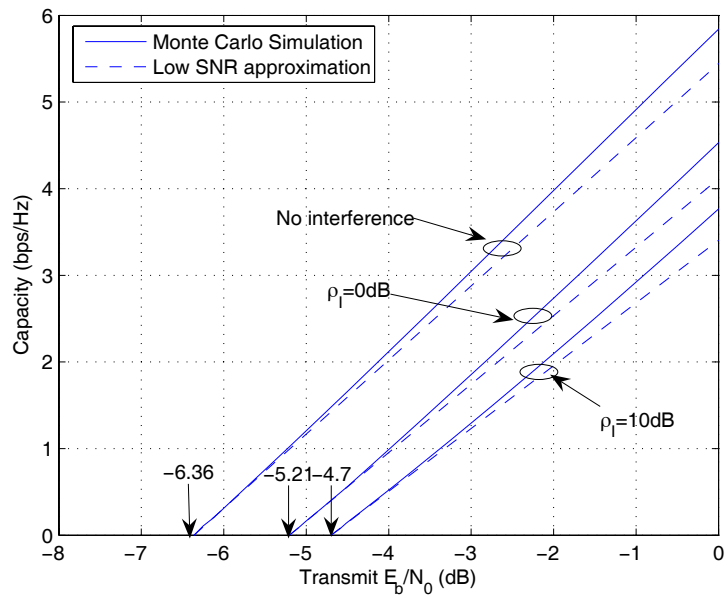

Fig. 2. Low SNR capacity versus transmit $E_{b} / N_{0}$ for Rician fading channels with different $\rho_{I}$ when $K=1, N_{t}=2$ and $N_{r}=3$.

Fig. 1 investigates the impact of $N_{r}$ on $E_{b} / N_{0 \text { min }}$. To isolate the effect of $N_{r}$, we have set $K=0$ to eliminate the possible impact from channel mean matrix $\mathbf{H}_{0}$. From the results of Fig. 1, it can be seen that the increase of $N_{r}$ helps to reduce the required $E_{b} / N_{0 \text { min }}$, which confirms the analysis of Corollary 1. Moreover, we observe that when $N_{r}$ increases, so does the wideband slope $S_{0}$, which indicates the double benefits of increasing $N_{r}$. In addition, when compared with the Monte-Carlo simulation results, the analytical results show very high accuracy in terms of $E_{b} / N_{0 \text { min }}$, and also the wideband slope $S_{0}$ if the SNR of interest is sufficiently low, i.e., below $2 \mathrm{bps} / \mathrm{Hz}$ of capacity.

In Fig. 2, results for the low SNR capacity approximation are plotted for $3 \times 2$ MIMO Rician channels with $K=1$.

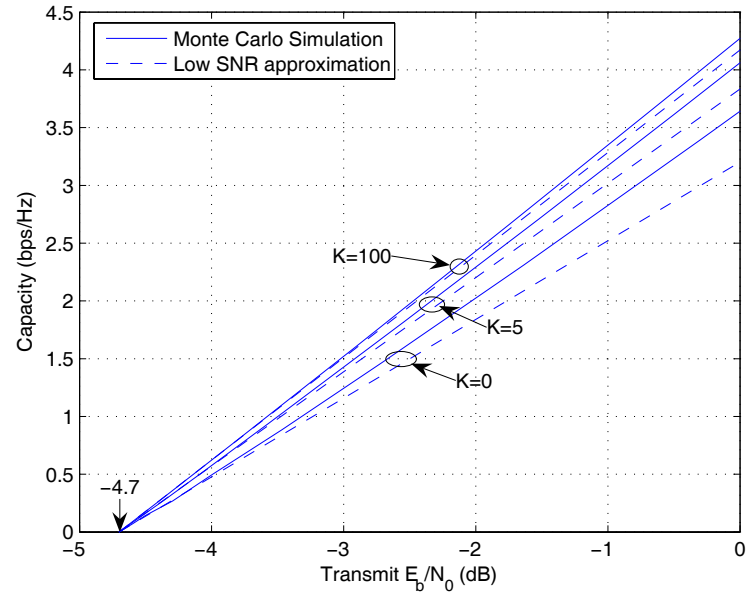

Fig. 3. Low SNR capacity versus transmit $E_{b} / N_{0}$ for various Rician factor $K$ when $N_{t}=2, N_{r}=3$ and $\rho_{I}=10 \mathrm{~dB}$.

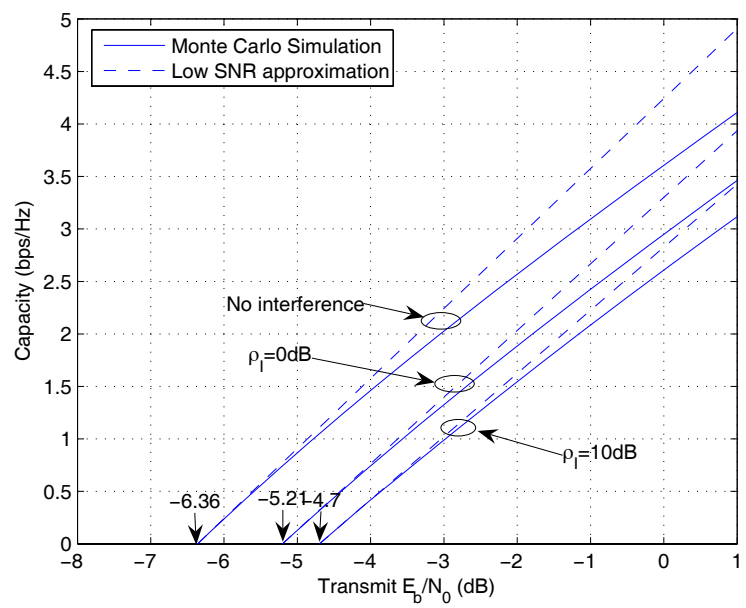

Fig. 4. Low SNR capacity versus transmit $E_{b} / N_{0}$ for rank1 mean Rician fading channels when $N_{t}=2, N_{r}=3$ and $K=100$.

Results reveal a good agreement between the analysis and the simulations. We also see that the increase in the interference power degrades the capacity performance by increasing the required $E_{b} / N_{0 \min }$, while the impact on $S_{0}$ is not so pronounced. Furthermore, the increase in $E_{b} / N_{0 \text { min }}$ from a channel without interference to that with a $10 \mathrm{~dB}$ of INR is about 0.1 , which appears to be very close to the upper bound we obtained in Corollary $1(\ln 2) /\left(N_{r}\left(N_{r}-1\right)\right)=0.115$.

Results in Fig. 3 are provided for the capacity of $3 \times 2$ MIMO Rician channels for different Rician- $K$ factors in the low SNR regime according to Theorem 1 . The curves indicate the accuracy of our analytical expression and that the range for a good approximation improves if $K$ increases. In particular, the approximation is very good for the capacity range from 


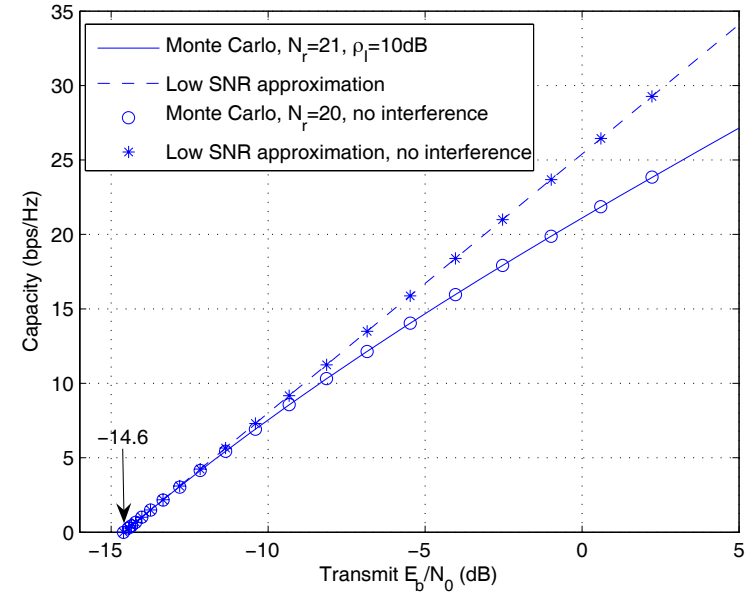

Fig. 5. Low SNR capacity versus transmit $E_{b} / N_{0}$ for Rayleigh fading channels when $N_{t}=3$ with different $N_{r}$ and $\rho_{I}$.

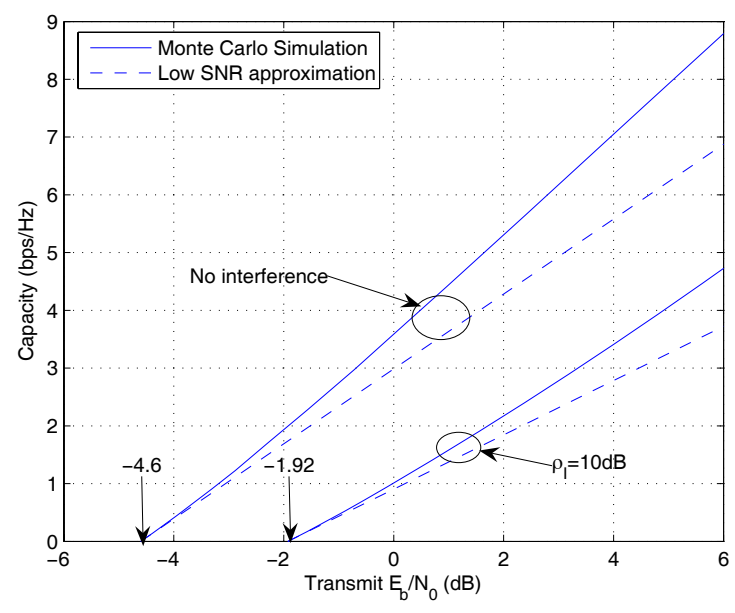

Fig. 6. Low SNR capacity versus transmit $E_{b} / N_{0}$ for Rayleigh-product channel when $N_{t}=3, N_{s}=6$ and $N_{r}=2$.

0 to 10 , when $K=100$. Also, results demonstrate that the Rician $K$ factor affects the capacity performance through the wideband slope $S_{0}$ but not the $E_{b} / N_{0 \text { min }}$, and more specifically, the wideband slope $S_{0}$ increases when $K$ becomes larger. However, the increase is not very substantial. On the other hand, Fig. 4 plots the results for rank-1 mean $3 \times 2$ MIMO Rician channels in the large $K$ regime both with and without interference. Results confirm the correctness of the analytical results in Corollary 5.

In Fig. 5, we provide the results for MIMO Rayleigh fading channels. Two system configurations are investigated: one for $3 \times 21$ channels with a single interferer of $\rho_{I}=10 \mathrm{~dB}$, and the other for $3 \times 20$ channels without interference. As we can see, the results of the two systems almost overlap with inappreciable difference in the low SNR regime, which aligns with our analysis.

Results in Figs. 6 and 7 are provided for MIMO Rayleighproduct channels. Results show a good agreement between the analysis and simulations. We also observe that the level of

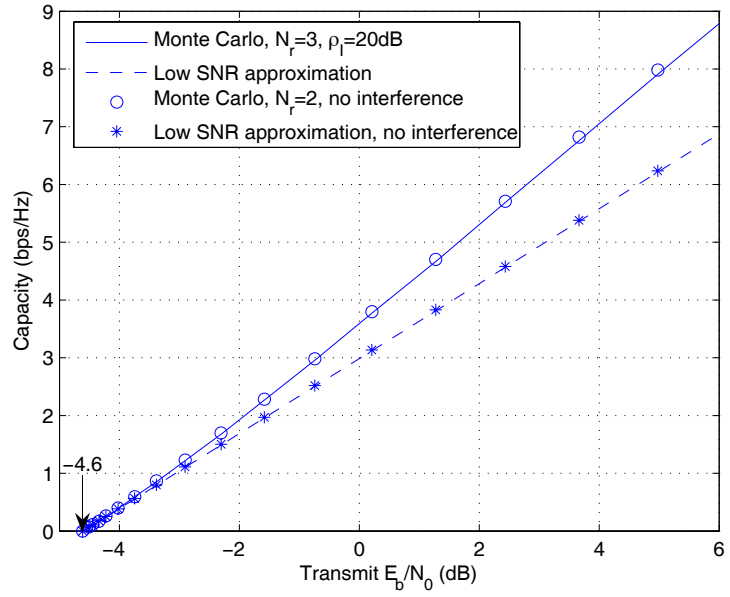

Fig. 7. Low SNR capacity versus transmit $E_{b} / N_{0}$ for Rayleigh-product channel when $N_{t}=2, N_{s}=6$ and different $N_{r}$ and $\rho_{I}$.

interference increases the required $E_{b} / N_{0 \min }$ and reduces the wideband slope $S_{0}$. On the other hand, Fig. 7 plots the results for two systems both with $N_{t}=2$ and $N_{s}=6$ : one with a single strong interferer of $\rho_{I}=20 \mathrm{~dB}$ and $N_{r}=3$, and the other with $N_{t}=2$ in an interference-free environment. Results for both systems overlap in the low SNR regime, which confirms our findings in (44) and (45).

\section{Conclusion}

This paper has studied the ergodic capacity of MIMO systems operating over Rician fading and Rayleigh-product channels in the presence of a single interferer and noise in the low SNR regime. Exact expressions for the minimum energy per information bit, $E_{b} / N_{0 \text { min }}$, and the wideband slope, $S_{0}$, were derived for both channels, which provide a much efficient way to evaluate the ergodic capacity of the system at low SNR as compared to the Monte-Carlo simulation method.

Based on the analytical expressions derived, we showed that for both MIMO Rician fading and Rayleigh-product channels, interference is detrimental in terms of ergodic capacity. It degrades the capacity performance by increasing $E_{b} / N_{0 \text { min }}$ and reducing $S_{0}$. However, the capacity deterioration due to interference is bounded. Specifically, the capacity of MIMO systems with $N_{r}$ receiving antennas in the presence of a single interferer is no worse than that of MIMO systems with $N_{r}-1$ receiving antennas in interference-free environment, regardless of the interference power level.

A number of additional insights on the impact of various system parameters have been observed. For MIMO Rician channels, we showed that both the structure of mean matrix and Rician factor $K$ will affect the ergodic capacity. Moreover, in the MISO case, we proved that a larger $K$ does not necessarily lead to an improvement on the capacity. In fact, how the Rician factor $K$ affects the capacity is closely related to the interference level $\rho_{I}$, which is very different to the case without interference, where it has been shown that a larger $K$ increases the capacity. For MIMO Rayleigh-product channels, we revealed that the number of scatterers has a positive effect 
on the ergodic capacity, i.e., when $N_{s}$ increases, the ergodic capacity improves. Furthermore, in the presence of a single interferer, the capacity of MIMO Rayleigh-product channels is upper bounded by the capacity of a MIMO Rayleigh fading channel with the same number of transmit and receive antennas.

To make the problem tractable, we have adopted the single interferer assumption, which has permitted the derivation of closed-form analytical expressions and a number of important physical insights. Nevertheless, the assumption has made the results less general and a more thorough investigation of the general scenario with multiple interferers is left for future work.

\section{APPENDIX A}

\section{SOME STATISTICAL RESUlTS}

Lemma 1: For any $m \times 1$ vector $\mathbf{h} \sim \mathcal{C N}(\mathbf{0}, \mathbf{I})$, and positive number $t$, let $\boldsymbol{\Lambda} \triangleq\left(t \mathbf{h h}^{\dagger}+\mathbf{I}\right)^{-1}$. Then,

$$
\begin{aligned}
\mathrm{E}\{\operatorname{tr}\{\boldsymbol{\Lambda}\}\} & =m-1+D_{1}(m, t), \\
\mathrm{E}\left\{\operatorname{tr}\left\{\boldsymbol{\Lambda}^{2}\right\}\right\} & =m-1+D_{2}(m, t), \\
\mathrm{E}\left\{\operatorname{tr}^{2}\{\boldsymbol{\Lambda}\}\right\} & =(m-1)^{2}+2(m-1) D_{1}(m, t)+D_{2}(m, t) .
\end{aligned}
$$

Proof: The result can be obtained by noticing the unitary invariant of vector $\mathbf{h}$, and using the integration formula [30, (3.385.5)].

Lemma 2: For any $m \times n$ matrix $\mathbf{H} \sim \mathcal{C N}(\mathbf{0}, \mathbf{I} \otimes \mathbf{I}), m \times 1$ vector $\mathbf{h} \sim \mathcal{C N}(\mathbf{0}, \mathbf{I})$, and positive constant $t$, we have

$$
\begin{aligned}
& \mathrm{E}\left\{\operatorname{tr}\left\{\mathbf{H}^{\dagger} \boldsymbol{\Lambda} \mathbf{H}\right\}\right\}=n(m-1)+n D_{1}(m, t), \\
& \mathrm{E}\left\{\operatorname{tr}\left\{\left(\mathbf{H}^{\dagger} \boldsymbol{\Lambda} \mathbf{H}\right)^{2}\right\}\right\}=n(m-1)(n+m-1)+ \\
& \left(n^{2}+n\right) D_{2}(m, t)+2 n(m-1) D_{1}(m, t), \\
& \mathrm{E}\left\{\operatorname{tr}^{2}\left\{\mathbf{H}^{\dagger} \boldsymbol{\Lambda} \mathbf{H}\right\}\right\}=n(m-1)(m n-n+1)+ \\
& \left(n^{2}+n\right) D_{2}(m, t)+2(m-1) n^{2} D_{1}(m, t),
\end{aligned}
$$

where $\boldsymbol{\Lambda}$ has been defined in Lemma 1 .

Proof: Utilizing the unitary invariant property of the distributions of $\mathbf{H}$ and $\mathbf{h}$, conditioned on $\boldsymbol{\Lambda}$, we have

$$
\begin{aligned}
& \mathrm{E}\left\{\operatorname{tr}\left\{\mathbf{H}^{\dagger} \boldsymbol{\Lambda} \mathbf{H}\right\} \mid \boldsymbol{\Lambda}\right\}=\mathrm{E}\left\{\operatorname{tr}\left\{\mathbf{H}^{\dagger} \boldsymbol{\Lambda} \mathbf{H}\right\} \mid \boldsymbol{\Lambda}\right\} \\
& \left.=\mathrm{E}\left\{(\operatorname{vec}(\mathbf{H}))^{\dagger}(\mathbf{I} \otimes \boldsymbol{\Lambda}) \operatorname{vec}(\mathbf{H})\right\} \mid \boldsymbol{\Lambda}\right\}=n \operatorname{tr}(\boldsymbol{\Lambda}) .
\end{aligned}
$$

Similarly, conditioned on $\boldsymbol{\Lambda}, \mathrm{E}\left\{\operatorname{tr}\left\{\left(\mathbf{H}^{\dagger} \boldsymbol{\Lambda} \mathbf{H}\right)^{2}\right\}\right\}$ can be expressed as

$$
\begin{aligned}
& \mathrm{E}\left\{\operatorname{tr}\left\{\left(\mathbf{H}^{\dagger} \boldsymbol{\Lambda} \mathbf{H}\right)^{2}\right\} \mid \boldsymbol{\Lambda}\right\}=\mathrm{E}\left\{\operatorname{tr}\left\{\left(\mathbf{H}^{\dagger} \boldsymbol{\Lambda} \mathbf{H}\right)^{2}\right\} \mid \boldsymbol{\Lambda}\right\} \\
& =\operatorname{tr}^{2}\{\mathbf{I}\} \operatorname{tr}\left\{\boldsymbol{\Lambda}^{2}\right\}+\operatorname{tr}^{2}\{\boldsymbol{\Lambda}\} \operatorname{tr}\{\mathbf{I}\},
\end{aligned}
$$

where (55) comes from [32, Lemma 6]. Finally, conditioned on $\Lambda$, and with the help of [32, Lemma 5], we have

$$
\mathrm{E}\left\{\operatorname{tr}^{2}\left\{\mathbf{H}^{\dagger} \boldsymbol{\Lambda} \mathbf{H}\right\} \mid \boldsymbol{\Lambda}\right\}=\operatorname{tr}\left\{\mathbf{I}^{2}\right\} \operatorname{tr}\left\{\boldsymbol{\Lambda}^{2}\right\}+\operatorname{tr}^{2}\{\mathbf{I}\} \operatorname{tr}^{2}\{\boldsymbol{\Lambda}\} .
$$

The desired results can be obtained by taking expectation on $\Lambda$ with the help of Lemma 1 .

Lemma 3: When $m \rightarrow \infty$ or $t \rightarrow \infty$, we have $D_{1}(m, t)=$ $D_{2}(m, t)=0$. On the other hand, if $t \rightarrow 0$, then $D_{1}(m, t)=$ $D_{2}(m, t)=1$.

Proof: First, we note that the confluent hypergeometric function can be expressed in terms of exponential integral function $E_{n}(\cdot)$ [34], so that

$$
\Psi\left(m, m, \frac{1}{t}\right)=t^{m-1} e^{\frac{1}{t}} E_{m}\left(\frac{1}{t}\right),
$$

and

$$
\begin{aligned}
& \Psi\left(m, m-1, \frac{1}{t}\right) \\
& \quad=\frac{t^{m-2}}{m-1}\left[e^{\frac{1}{t}} E_{m-1}\left(\frac{1}{t}\right)\left(m-1+\frac{1}{t}\right)-1\right] .
\end{aligned}
$$

Moreover, the exponential integral function satisfies the following inequality [35, (5.1.19)]

$$
\frac{1}{x+n}<e^{x} E_{n}(x) \leq \frac{1}{x+n-1}, \text { for } x>0 .
$$

Then, we can establish the following two inequalities:

$$
\begin{aligned}
\frac{1}{1+t m} & <D_{1}(m, t) \leq \frac{1}{1+t(m-1)}, \\
0 & <D_{2}(m, t) \leq \frac{1}{t(m-1)[t(m-2)-1]} .
\end{aligned}
$$

For the cases $m \rightarrow \infty$ and $t \rightarrow \infty$, it is easy to see that both sides of (60) and (61) approach 0 and therefore, we have $D_{1}(m, t)=D_{2}(m, t)=0$. Now, consider the case $t \rightarrow 0$. It is easily observed that both sides of (60) will approach 1 and hence, $D_{1}(m, t)=1$. However, since $\frac{1}{t(m-1)[t(m-2)-1]} \rightarrow \infty$ when $t \rightarrow 0$, the two sides of (61) diverge. To obtain the limit of $D_{2}(m, t)$, define $a \triangleq \frac{1}{t}$. Utilizing the property of confluent hypergeometric function [35, (13.4.24)] and [35, (13.4.21)], we have

$$
\begin{aligned}
a^{m} \Psi(m, m-1, a)= & (1-m) a^{m} \Psi(m, m, a)+ \\
& m a^{m+1} \Psi(m+1, m+1, a) .
\end{aligned}
$$

Then, from (60), we have

$$
a^{m} \Psi(m, m, a)=1, \text { as } a \rightarrow \infty .
$$

Therefore, (62) reduces to

$$
a^{m} \Psi(m, m-1, a)=(1-m)+m=1, \text { as } a \rightarrow \infty,
$$

which completes the proof.

\section{APPENDIX B}

\section{PROOF OF THEOREM 1}

With the help of the following determinant properties,

$$
\begin{gathered}
\left.\frac{d}{d x} \ln \operatorname{det}(\mathbf{I}+x \mathbf{A})\right|_{x=0}=\operatorname{tr}\{\mathbf{A}\}, \\
\left.\frac{d^{2}}{d^{2} x} \ln \operatorname{det}(\mathbf{I}+x \mathbf{A})\right|_{x=0}=-\operatorname{tr}\left\{\mathbf{A}^{2}\right\},
\end{gathered}
$$

and treating $\frac{\mathbf{H}^{\dagger}\left(\rho_{I} \mathbf{h} \mathbf{h}^{\dagger}\right)^{-1} \mathbf{H}}{\frac{1}{N_{r}} E\left\{\operatorname{tr}\left\{\mathbf{H}^{\dagger}\left(\rho_{I} \mathbf{h h}^{\dagger}\right)^{-1} \mathbf{H}\right\}\right\}}$ as one matrix, as well as noticing that the order of expectation operation on $\mathbf{H}$ and $\mathbf{h}$ and the derivative operation on $\rho$ can be exchanged, we compute the first and second derivatives of $C(\rho)$ at $\rho=0$ as

$$
\begin{aligned}
& \dot{C}(0)=N_{r} \log _{2} e, \\
& \ddot{C}(0)=-\frac{N_{r}^{2} \mathrm{E}\left\{\operatorname{tr}\left\{\left(\mathbf{H}^{\dagger} \boldsymbol{\Lambda} \mathbf{H}\right)^{2}\right\}\right\} \log _{2} e}{\mathrm{E}^{2}\left\{\operatorname{tr}\left\{\mathbf{H}^{\dagger} \boldsymbol{\Lambda} \mathbf{H}\right\}\right\}} .
\end{aligned}
$$


As a result, $\frac{E_{b}}{N_{0}} \min$ and $S_{0}$ can be computed according to (9) as

$$
\begin{aligned}
{\frac{E_{b}}{N_{0} \text { min }}} & =\frac{N_{t} \ln 2}{\mathrm{E}\left\{\operatorname{tr}\left\{\mathbf{H}^{\dagger} \boldsymbol{\Lambda} \mathbf{H}\right\}\right\}}, \\
S_{0} & =\frac{2 \mathrm{E}^{2}\left\{\operatorname{tr}\left\{\mathbf{H}^{\dagger} \boldsymbol{\Lambda} \mathbf{H}\right\}\right\}}{\mathrm{E}\left\{\operatorname{tr}\left\{\left(\mathbf{H}^{\dagger} \boldsymbol{\Lambda} \mathbf{H}\right)^{2}\right\}\right\}} .
\end{aligned}
$$

To proceed, we need to compute $\mathrm{E}\left\{\operatorname{tr}\left\{\mathbf{H}^{\dagger} \boldsymbol{\Lambda} \mathbf{H}\right\}\right\}$ and $\mathrm{E}\left\{\operatorname{tr}\left\{\left(\mathbf{H}^{\dagger} \boldsymbol{\Lambda} \mathbf{H}\right)^{2}\right\}\right\}$.

Following similar steps as in [19], we have

$$
\begin{array}{r}
\mathrm{E}\left\{\operatorname{tr}\left\{\mathbf{H}^{\dagger} \boldsymbol{\Lambda} \mathbf{H}\right\}\right\}=\frac{K}{K+1} \mathrm{E}\left\{\operatorname{tr}\left\{\mathbf{H}_{0} \mathbf{H}_{0}^{\dagger} \boldsymbol{\Lambda}\right\}\right\}+ \\
\frac{1}{K+1} \mathrm{E}\left\{\operatorname{tr}\left\{\mathbf{H}_{w} \mathbf{H}_{w}^{\dagger} \boldsymbol{\Lambda}\right\}\right\} .
\end{array}
$$

The first term of (71) can be computed with the help of [19, Lemma 3] as

$$
\begin{array}{r}
\frac{K}{K+1} \mathrm{E}\left\{\operatorname{tr}\left\{\mathbf{H}_{0} \mathbf{H}_{0}^{\dagger} \boldsymbol{\Lambda}\right\}\right\}=\frac{K \operatorname{tr}\left\{\mathbf{H}_{0} \mathbf{H}_{0}^{\dagger}\right\} \mathrm{E}\{\operatorname{tr}\{\boldsymbol{\Lambda}\}\}}{(K+1) N_{r}} \\
=\frac{K N_{t}}{K+1}\left[D_{1}\left(N_{r}, \rho_{I}\right)+N_{r}-1\right],
\end{array}
$$

where in (72), we have used the fact that $\operatorname{tr}\left\{\mathbf{H}_{0} \mathbf{H}_{0}^{\dagger}\right\}=N_{t} N_{r}$ and the result of Lemma 1. On the other hand, the second term of (71) can be obtained directly from Lemma 2. As such,

$$
\mathrm{E}\left\{\operatorname{tr}\left\{\mathbf{H}^{\dagger} \boldsymbol{\Lambda} \mathbf{H}\right\}\right\}=N_{t}\left[D_{1}\left(N_{r}, \rho_{I}\right)+N_{r}-1\right] .
$$

Now, it remains to derive the expression for $\mathrm{E}\left\{\operatorname{tr}\left\{\left(\mathbf{H}^{\dagger} \boldsymbol{\Lambda} \mathbf{H}\right)^{2}\right\}\right\}$. Utilizing the zero mean property of $\mathbf{H}_{w}$ and after some basic algebraic manipulations, we express $\mathrm{E}\left\{\operatorname{tr}\left\{\left(\mathbf{H}^{\dagger} \boldsymbol{\Lambda} \mathbf{H}\right)^{2}\right\}\right\}$ as in Eq. (74) shown on the top of this page. The first term can be easily obtained directly from Lemma 2. Therefore, here, we focus on the last three terms. With the help of [19, Lemma 3], we compute the second term as

$$
\begin{aligned}
& \mathrm{E}\left\{\operatorname{tr}\left\{\left(\mathbf{H}_{0} \mathbf{H}_{0}^{\dagger} \boldsymbol{\Lambda}\right)^{2}\right\}\right\}= \\
& \frac{\operatorname{tr}\left\{\left(\mathbf{H}_{0} \mathbf{H}_{0}^{\dagger}\right)^{2}\right\}}{N_{r}^{2}-1}\left(\mathrm{E}\left\{\operatorname{tr}^{2}\{\boldsymbol{\Lambda}\}\right\}-\frac{1}{N_{r}} \mathrm{E}\left\{\operatorname{tr}\left\{\boldsymbol{\Lambda}^{2}\right\}\right\}\right) \\
& +\frac{N_{t}^{2} N_{r}^{2}}{N_{r}^{2}-1}\left(\mathrm{E}\left\{\operatorname{tr}\left\{\boldsymbol{\Lambda}^{2}\right\}\right\}-\frac{1}{N_{r}} \mathrm{E}\left\{\operatorname{tr}^{2}\{\boldsymbol{\Lambda}\}\right\}\right) .
\end{aligned}
$$

Similarly, the third and fourth terms can be obtained as follows:

$$
\begin{aligned}
& \mathrm{E}\left\{\operatorname{tr}\left\{\mathbf{H}_{w} \mathbf{H}_{w}^{\dagger} \boldsymbol{\Lambda} \mathbf{H}_{0} \mathbf{H}_{0}^{\dagger} \boldsymbol{\Lambda}\right\}\right\} \\
& =\frac{1}{N_{r}} \mathrm{E}\left\{\operatorname{tr}\left\{\mathbf{H}_{w} \mathbf{H}_{w}^{\dagger}\right\}\right\} \mathrm{E}\left\{\operatorname{tr}\left\{\mathbf{H}_{0} \mathbf{H}_{0}^{\dagger} \boldsymbol{\Lambda}^{2}\right\}\right\} \\
& =N_{t} \mathrm{E}\left\{\operatorname{tr}\left\{\mathbf{H}_{0} \mathbf{H}_{0}^{\dagger} \boldsymbol{\Lambda}^{2}\right\}\right\}=N_{t}^{2} \mathrm{E}\left\{\operatorname{tr}\left\{\boldsymbol{\Lambda}^{2}\right\}\right\},
\end{aligned}
$$

and

$$
\begin{aligned}
& \mathrm{E}\left\{\operatorname{tr}\left\{\mathbf{H}_{w}^{\dagger} \boldsymbol{\Lambda} \mathbf{H}_{w} \mathbf{H}_{0}^{\dagger} \boldsymbol{\Lambda} \mathbf{H}_{0}\right\}\right\} \\
& =\frac{1}{N_{r}} \mathrm{E}\{\operatorname{tr}\{\boldsymbol{\Lambda}\}\} \mathrm{E}\left\{\operatorname{tr}\left\{\mathbf{H}_{w} \mathbf{H}_{0}^{\dagger} \boldsymbol{\Lambda} \mathbf{H}_{0} \mathbf{H}_{w}^{\dagger}\right\}\right\}
\end{aligned}
$$

$$
\begin{aligned}
& =\frac{\mathrm{E}\{\operatorname{tr}\{\boldsymbol{\Lambda}\}\}}{N_{t} N_{r}} \mathrm{E}\left\{\operatorname{tr}\left\{\mathbf{H}_{w} \mathbf{H}_{w}^{\dagger}\right\}\right\} \mathrm{E}\left\{\operatorname{tr}\left\{\mathbf{H}_{0}^{\dagger} \boldsymbol{\Lambda} \mathbf{H}_{0}\right\}\right\} \\
& =N_{t} \mathrm{E}^{2}\{\operatorname{tr}\{\boldsymbol{\Lambda}\}\} .
\end{aligned}
$$

As a result, $\mathrm{E}\left\{\operatorname{tr}\left\{\left(\mathbf{H}^{\dagger} \boldsymbol{\Lambda} \mathbf{H}\right)^{2}\right\}\right\}$ can be computed as shown on the top of next page. Finally, applying Lemma 1 yields the desired result.

\section{APPENDIX C \\ PROOF OF COROLLARY 1}

Define the function $f\left(N_{r}\right) \triangleq N_{r}-1+A_{0}$. Hence, we are required to prove that $f\left(N_{r}\right)$ is an increasing function of $N_{r}$ which we do by considering

$$
f\left(N_{r}+1\right)-f\left(N_{r}\right)=1+D_{1}\left(N_{r}+1, \rho_{I}\right)-D_{1}\left(N_{r}, \rho_{I}\right) .
$$

With the help of (60), we can bound $f\left(N_{r}+1\right)-f\left(N_{r}\right) \geq 0$ by

$$
\begin{aligned}
& f\left(N_{r}+1\right)-f\left(N_{r}\right)>1+\frac{1}{1+\rho_{I}\left(N_{r}+1\right)}-\frac{1}{1+\rho_{I}\left(N_{r}-1\right)} \\
& =1-\frac{2 \rho_{I}}{\left(1+\rho_{I} N_{r}\right)^{2}-\rho_{I}^{2}} \geq 1-\frac{2 \rho_{I}}{1+2 \rho_{I}}>0,
\end{aligned}
$$

which completes the first half of the proof.

To prove the corresponding part for $\rho_{I}$, we define another function $g\left(\rho_{I}\right)$ as $g\left(\rho_{I}\right)=D_{1}\left(N_{r}, \rho_{I}\right)$ and then show that $g\left(\rho_{I}\right)$ is monotonically decreasing. To do so, we compute the first derivative of $g\left(\rho_{I}\right)$ with the help of the derivative formula of a confluent hypergeometric function [35, (13.4.20)]

$$
\begin{aligned}
g^{\prime}\left(\rho_{I}\right) & =N_{r} \rho_{I}^{-N_{r}-2} \Psi\left(N_{r}+1, N_{r}+1, \rho_{I}^{-1}\right) \\
& -N_{r} \rho_{I}^{-N_{r}-1} \Psi\left(N_{r}, N_{r}, \rho_{I}^{-1}\right) \\
& <N_{r} \rho_{I}^{-1} \frac{1}{1+\rho_{I} N_{r}}-N_{r} \rho_{I}^{-1} \frac{1}{1+\rho_{I} N_{r}}=0 .
\end{aligned}
$$

Hence, $g\left(\rho_{I}\right)$ is a monotonic decreasing function. Therefore, we have

$$
g\left(\rho_{I} \rightarrow \infty\right) \leq g\left(\rho_{I}\right) \leq g\left(\rho_{I} \rightarrow 0\right) .
$$

With the help of Lemma 3, we have $g\left(\rho_{I} \rightarrow 0\right)=1$ and $g\left(\rho_{I} \rightarrow \infty\right)=0$. As a consequence, the increase in $E_{b} / N_{0 \text { min }}$ is bounded by

$$
\begin{aligned}
& \frac{\ln 2}{N_{r}-1+g\left(\rho_{I} \rightarrow \infty\right)}-\frac{\ln 2}{N_{r}-1+g\left(\rho_{I} \rightarrow 0\right)} \\
& =\ln 2\left(\frac{1}{N_{r}}-\frac{1}{N_{r}-1}\right)=\frac{\ln 2}{N_{r}\left(N_{r}-1\right)},
\end{aligned}
$$

which completes the proof.

\section{APPENDIX D}

\section{PROOF OF COROLLARY 4}

The first derivative of $S_{0}$ with respect to $N_{t}$ can be obtained as

$$
S_{0}^{\prime}\left(N_{t}\right)=\frac{2(K+1)^{2} D_{1}\left(1, \rho_{I}\right)^{2}\left(2 K+D_{2}\left(1, \rho_{I}\right)\right)}{\left(2 K+\left(1+N_{t}(K+1)^{2} D_{2}\left(1, \rho_{I}\right)\right)\right)^{2}} \geq 0,
$$

which has proved the first claim. Similarly, the first derivative of $S_{0}$ with respect to $K$ is given by

$$
S_{0}^{\prime}(K)=\frac{4 N_{t}(K+1) D_{1}\left(1, \rho_{I}\right)^{2}\left(K+D_{2}\left(1, \rho_{I}\right)-1\right)}{\left(2 K+\left(1+N_{t}(K+1)^{2} D_{2}\left(1, \rho_{I}\right)\right)\right)^{2}} .
$$




$$
\begin{aligned}
\mathrm{E}\left\{\operatorname{tr}\left\{\left(\mathbf{H}^{\dagger} \boldsymbol{\Lambda} \mathbf{H}\right)^{2}\right\}\right\}=\frac{1}{(K+1)^{2}} \mathrm{E}\left\{\operatorname{tr}\left\{\left(\mathbf{H}_{w} \mathbf{H}_{w}^{\dagger} \boldsymbol{\Lambda}\right)^{2}\right\}\right\}+\frac{K^{2}}{(K+1)^{2}} \mathrm{E}\left\{\operatorname{tr}\left\{\left(\mathbf{H}_{0} \mathbf{H}_{0}^{\dagger} \boldsymbol{\Lambda}\right)^{2}\right\}\right\} \\
+\frac{2 K}{(K+1)^{2}}\left(\mathrm{E}\left\{\operatorname{tr}\left\{\mathbf{H}_{w} \mathbf{H}_{w}^{\dagger} \boldsymbol{\Lambda} \mathbf{H}_{0} \mathbf{H}_{0}^{\dagger} \boldsymbol{\Lambda}\right\}\right\}+\mathrm{E}\left\{\operatorname{tr}\left\{\mathbf{H}_{w}^{\dagger} \boldsymbol{\Lambda} \mathbf{H}_{w} \mathbf{H}_{0}^{\dagger} \boldsymbol{\Lambda} \mathbf{H}_{0}\right\}\right\}\right) .
\end{aligned}
$$

$$
\begin{aligned}
& \mathrm{E}\left\{\operatorname{tr}\left\{\left(\mathbf{H}^{\dagger} \boldsymbol{\Lambda} \mathbf{H}\right)^{2}\right\}\right\}=\frac{1}{(K+1)^{2}}\left[\frac{K^{2}\left(\operatorname{tr}\left\{\left(\mathbf{H}_{0} \mathbf{H}_{0}^{\dagger}\right)^{2}\right\}-N_{t}^{2} N_{r}\right)}{N_{r}^{2}-1}+N_{t}\right] \mathrm{E}\left\{\operatorname{tr}^{2}\{\boldsymbol{\Lambda}\}\right\} \\
& +\frac{1}{(K+1)^{2}}\left[\frac{K^{2}\left(N_{t}^{2} N_{r}^{3}-\operatorname{tr}\left\{\left(\mathbf{H}_{0} \mathbf{H}_{0}^{\dagger}\right)^{2}\right\}\right)}{N_{r}\left(N_{r}^{2}-1\right)}+(1+2 K) N_{t}^{2}\right] \operatorname{E}\left\{\operatorname{tr}\left\{\boldsymbol{\Lambda}^{2}\right\}\right\}+\frac{2 K N_{t}}{(K+1)^{2}} \mathrm{E}^{2}\{\operatorname{tr}\{\boldsymbol{\Lambda}\}\} .
\end{aligned}
$$

To complete the proof, we further have

$$
\begin{aligned}
& D_{2}\left(1, \rho_{I}\right)=\frac{1}{\rho_{I}} \int_{0}^{\infty} e^{-\frac{1}{\rho_{I}} x}(1+x)^{-2} d x= \\
& \quad \int_{0}^{\infty} e^{-x}\left(1+\rho_{I} x\right)^{-2} d x \leq \int_{0}^{\infty} e^{-x} d x=1 .
\end{aligned}
$$

Because of the fact that $0 \leq D_{2}\left(1, \rho_{I}\right) \leq 1$, we have $S_{0}^{\prime}(K)>$ 0 if $0 \leq K<1-D_{2}\left(1, \rho_{I}\right)$ and $S_{0}^{\prime}(K) \leq 0$ if $K \geq 1-$ $D_{2}\left(1, \rho_{I}\right)$, which has proved the second claim.

\section{APPENDIX E \\ PROOF OF THEOREM 2}

Following the same steps as in the proof of Theorem 1, for MIMO Rayleigh-product channels, it can be derived that

$$
\begin{aligned}
\frac{E_{b}}{N_{0 \text { min }}}= & \frac{N_{t} \ln 2}{\mathrm{E}\left\{\frac{1}{N_{s}} \operatorname{tr}\left\{\mathbf{H}_{2}^{\dagger} \mathbf{H}_{1}^{\dagger} \mathbf{\Lambda} \mathbf{H}_{1} \mathbf{H}_{2}\right\}\right\}}, \\
S_{0} & =\frac{2 \mathrm{E}^{2}\left\{\frac{1}{N_{s}} \operatorname{tr}\left\{\mathbf{H}_{2}^{\dagger} \mathbf{H}_{1}^{\dagger} \boldsymbol{\Lambda} \mathbf{H}_{1} \mathbf{H}_{2}\right\}\right\}}{\mathrm{E}\left\{\frac{1}{N_{s}^{2}} \operatorname{tr}\left\{\left(\mathbf{H}_{2}^{\dagger} \mathbf{H}_{1}^{\dagger} \boldsymbol{\Lambda} \mathbf{H}_{1} \mathbf{H}_{2}\right)^{2}\right\}\right\}} .
\end{aligned}
$$

First defining $\mathbf{W} \triangleq \mathbf{H}_{1}^{\dagger} \boldsymbol{\Lambda} \mathbf{H}_{1}$, conditioned on $\mathbf{W}$, we get

$$
\begin{aligned}
\mathrm{E}\left\{\frac{1}{N_{s}} \operatorname{tr}\right. & \left.\left\{\mathbf{H}_{2}^{\dagger} \mathbf{H}_{1}^{\dagger} \boldsymbol{\Lambda} \mathbf{H}_{1} \mathbf{H}_{2}\right\} \mid \mathbf{W}\right\}= \\
& \frac{1}{N_{s}} \mathrm{E}\left\{\operatorname{tr}\left\{\mathbf{H}_{2}^{\dagger} \mathbf{W} \mathbf{H}_{2}\right\} \mid \mathbf{W}\right\}=\frac{N_{t}}{N_{s}} \operatorname{tr}\{\mathbf{W}\} .
\end{aligned}
$$

Similarly, we have

$$
\begin{aligned}
& \mathrm{E}\left\{\frac{1}{N_{s}^{2}} \operatorname{tr}\left\{\left(\mathbf{H}_{2}^{\dagger} \mathbf{H}_{1}^{\dagger} \mathbf{\Lambda} \mathbf{H}_{1} \mathbf{H}_{2}\right)^{2}\right\} \mid \mathbf{W}\right\} \\
& =\frac{1}{N_{s}^{2}} \mathrm{E}\left\{\operatorname{tr}\left\{\left(\mathbf{H}_{2}^{\dagger} \mathbf{W} \mathbf{H}_{2}\right)^{2}\right\} \mid \mathbf{W}\right\} \\
& =\frac{1}{N_{s}^{2}}\left(\operatorname{tr}\{\mathbf{I}\}^{2} \operatorname{tr}\left\{\mathbf{W}^{2}\right\}+\operatorname{tr}^{2}\{\mathbf{W}\} \operatorname{tr}\{\mathbf{I}\}\right) \\
& =\frac{1}{N_{s}^{2}}\left(N_{t}^{2} \operatorname{tr}\left\{\mathbf{W}^{2}\right\}+N_{t} \operatorname{tr}^{2}\{\mathbf{W}\}\right) .
\end{aligned}
$$

With the help of Lemma 2 and further taking expectation on W in (93) and (96), the desired result can be obtained after some basic algebraic manipulations.

\section{APPENDIX F \\ PROOF OF COROLLARY 7}

Starting from Theorem 2, we rewrite $S_{0}$ as

$$
S_{0}=\frac{2 N_{t}}{\left(N_{t}+B_{2}\right) / N_{s}+1} .
$$

To this end, extract the terms inside $B_{2}$ without $N_{s}$ and simplify, which leads to

$$
S_{0}=\frac{2 N_{t}}{C_{1} / N_{s}+1+C_{2}},
$$

where $C_{2}$ is a constant and $C_{1}$ is given by

$$
\begin{aligned}
C_{1}=\frac{N_{t}\left(N_{r}-1\right)^{2}+}{N_{r}-1+2 D_{1}\left(N_{r}, \rho_{I}\right)\left(N_{r}-1\right) N_{t}} \\
\left(N_{r}-1+D_{1}\left(N_{r}, \rho_{I}\right)\right)^{2} \\
+\frac{D_{2}\left(N_{r}, \rho_{I}\right)\left(N_{t}+1\right)}{\left(N_{r}-1+D_{1}\left(N_{r}, \rho_{I}\right)\right)^{2}} .
\end{aligned}
$$

It is easy to observe that $C_{1}$ is a positive number, which proves the first part of the corollary. As for the limiting part, one can easily show that $\left.\frac{B_{2}}{N_{s}}\right|_{N_{s} \rightarrow \infty}=B_{1}$, which completes the proof.

\section{REFERENCES}

[1] J. H. Winters, "On the capacity of radio communication systems with diversity in a Rayleigh fading environment," IEEE J. Sel. Areas Commun., vol. SAC-5, no. 5, pp. 871-878, May 1987.

[2] I. E. Telatar, "Capacity of multi-antenna Gaussian channels," Euro. Trans. Telecommun. Related Tech., vol. 10, no. 6, pp. 585-596, Nov. 1999.

[3] G. J. Foschini and M. J. Gans, "On limits of wireless communication in a fading environment when using multiple antennas," Wireless Personal Commun., vol. 6, pp. 311-335, Mar. 1998.

[4] M. Chiani, M. Z. Win, and A. Zanella, "On the capacity of spatially correlated MIMO Rayleigh-fading channels," IEEE Trans. Inf. Theory, vol. 49, no. 10, pp. 2363-2371, Oct. 2003.

[5] P. J. Smith, S. Roy, and M. Shafi, "Capacity of MIMO systems with semicorrelated flat fading," IEEE Trans. Inf. Theory, vol. 49, no. 10, pp. 2781-2788, Oct. 2003.

[6] S. K. Jayaweera and H. V. Poor, "On the capacity of multiple-antenna systems in Rician fading," IEEE Trans. Wireless Commun., vol. 4, no. 3, pp. 1102-1111, May 2005.

[7] M. R. McKay and I. B. Collings, "General capacity bounds for spatially correlated Rician MIMO channels," IEEE Trans. Inf. Theory, vol. 51, no. 9, pp. 3121-3145, Sep. 2005.

[8] J. H. Winters, "Optimum combining in digital mobile radio with cochannel interference," IEEE Trans. Veh. Technol., vol. 33, pp. 144-155, Aug. 1984. 
[9] A. Shah, A. M. Haimovich, M. K. Simon, and M. S. Alouini, "Exact bit-error probability for optimum combining with a Rayleigh fading Gaussian cochannel interferer," IEEE Trans. Commun., vol. 48, no. 6, pp. 908-912, June 2000.

[10] R. S. Blum, "MIMO capacity with interference," IEEE J. Sel. Areas Commun., vol. 21, no. 5, pp. 793-801, June 2003.

[11] Y. Song and S. D. Blostein, "MIMO channel capacity in co-channel interference," in Proc. 21st Biennial Sym. Commun., Jan. 2002, pp. 220224, Kingston, Canada.

[12] M. Webb, M. Beach, and A. Nix, "Capacity limits of MIMO channels with co-channel interference," in Proc. IEEE Veh. Technol. Conf. Spring, May 2004, pp. 703-707, Milan, Italy.

[13] A. L. Moustakas, S. H. Simon, and A. M. Sengupta, "MIMO capacity throughput correlated channels in the presence of correlated interference and noise: a (not so) large $\mathrm{N}$ analysis," IEEE Trans. Inf. Theory, vol. 49, no. 10, pp. 2545-2561, Nov. 2003.

[14] M. Kang, L. Yang, and M. S. Alouini, "Capacity of MIMO channels in the presence of co-channel interference," Wireless Commun. Mob. Comput., no. 7, pp. 113-125, Mar. 2006.

[15] M. Chiani, M. Z. Win, and H. Shin, "MIMO networks: the effects of interference," IEEE Trans. Inf. Theory, vol. 56, no. 1, pp. 336-349, Jan. 2010.

[16] P. Bender, P. Black, M. Grob, R. Padovani, N. Sindhushyana, and S. Viterbi, "CDMA/HDR: a bandwidth efficient high speed wireless data service for nomadic users," IEEE Commun. Mag., vol. 38, no. 7, pp. 70-77, July 2000.

[17] 3rd Generation Partnership Project; Technical Specification Group Radio Access Network, "UTRA high speed downlink packet access," Tech. Rep., 3G TR 25.950, Mar. 2001.

[18] S. Verdú, "Spectral efficiency in the wideband regime," IEEE Trans. Inf. Theory, vol. 48, no. 6, pp. 1319-1343, June 2002.

[19] A. Lozano, A. M. Tulino, and S. Verdú, "Multiple-antenna capacity in the low-power regime," IEEE Trans. Inf. Theory, vol. 49, no. 10, pp. 2527-2544, Oct. 2003.

[20] A. M. Tulino, A. Lozano, and S. Verdu, "The impact of antenna correlation on the capacity of multiantenna channels," IEEE Trans. Inf. Theory, vol. 51, no. 7, pp. 2491-2509, July 2005.

[21] E. A. Jorswieck and H. Boche, "Multiple-antenna capacity in the lowpower regime: channel knowledge and correlation," in Proc. IEEE Int. Conf. Acoustic, Speech, Signal Process., 2005, pp. 385-388, Philadelphia, USA.

[22] W. Zhang and J. N. Laneman, "Benefits of spatial correlation for multiantenna non-coherent communication over fading channels at low SNR," IEEE Trans. Wireless Commun., vol. 6, no. 3, pp. 887-896, Mar. 2007.

[23] S. Jin, M. R. McKay, K. K. Wong, and X. Li, "Low SNR capacity of double-scattering MIMO channels with transmitter channel knowledge," in Proc. IEEE Int. Conf. Commun., Dresden, German, 2009.

[24] D. Gesbert, H. Bolcskei, D. A. Gore, and A. J. Paulraj, "Outdoor MIMO wireless channels: models and performance prediction," IEEE Trans. Commun., vol. 50, no. 12, pp. 1926-1934, Dec. 2002.

[25] P. Almers, F. Tufvesson, and A. F. Molisch, "Keyhole effect in MIMO wireless channels: measurements and theory," IEEE Trans. Wireless Commun., pp. 3596-3604, vol. 5, no. 12, Dec. 2006.

[26] D. Gunduz, A. Khojastepour, A. Goldsmith, and H. V. Poor, "Multihop MIMO relay networks: diversity-multiplexing trade-off analysis," submitted to IEEE Trans. Wireless Commun..

[27] S. Jin, M. McKay, C. Zhong, and K. Wong, "Ergodic capacity analysis of amplify-and-forward MIMO dual-hop systems," accepted in IEEE Trans. Inf. Theory.

[28] C. Zhong, S. Jin, and K. K. Wong, "MIMO Rayleigh-product channels with co-channel interference," IEEE Trans. Commun., vol. 57, no. 6, pp. 1824-1835, June 2009.

[29] F. R. Farrokhi, G. J. Foschini, A. Lozano, and R. Valenzuela, "Linkoptimal space-time processing with multiple transmit and receive antennas," IEEE Commun. Lett., vol. 5, no. 3, pp. 85-87, Mar. 2001.

[30] I. S. Gradshteyn and I. M. Ryzhik, Table of Integrals, Series, and Products, 5th edition. Orlando, FL: Academic Press, 1994.

[31] H. Bolcskei, M. Borgmann, and A. J. Paulraj, "Impact of the propagation environment on the performance of space-frequency coded MIMOOFDM," IEEE J. Sel. Areas Commun., vol. 21, no. 3, pp. 427-439, Apr. 2003.

[32] H. Shin and M. Z. Win, "MIMO diversity in the presence of double scattering," IEEE Trans. Inf. Theory, vol. 54, no. 7, pp. 2976-2996, July 2008.

[33] R. J. Murihead, Aspects of Multivariate Statistical Theory. Wiley Interscience, 1982.

[34] [Online]. Available: http://functions.wolfram.com.
[35] M. Abramowitz and I. A. Stegun, Handbook of Mathematical Functions. New York: Dover Publications Inc., 1974.

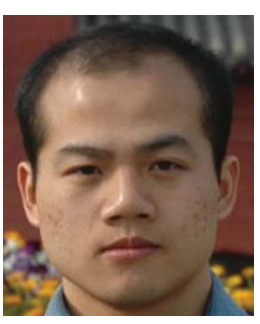

Caijun Zhong (S'07) Caijun Zhong received the B.S. degree in Information Engineering from the Xi' an Jiaotong University, Xi'an, China, in 2004, and the M.S. degree in Information Security in 2006, $\mathrm{Ph} . \mathrm{D}$. degree in Telecommunications in 2010, both from University College London, London, United Kingdom. He is currently a research fellow at the Institute for Electronics, Communications and Information Technologies (ECIT), Queen's University Belfast, Belfast, UK. His research interests include multivariate statistical theory, MIMO communications systems, cooperative communications.

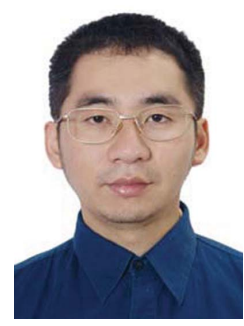

Shi Jin (S'06-M'07) Shi Jin received the B.S. degree in Communication Engineering from the Guilin University of Electronic Technology, Guilin, China, in 1996, and the M.S. degree from the Nanjing University of Posts \& Telecommunications, Nanjing, China, in 2003. He received the Ph.D. degree in Communication \& Information System at the Southeast University, China, in 2007. He then joined the National Mobile Communications Research Laboratory, Southeast University. From June 2007 to October 2009, he was also a Research Fellow in Adastral Park Research Campus at University College London, UK. His research interests include space-time wireless communications, random matrix theory, and information theory.

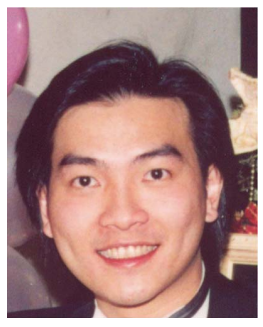

Kai-Kit Wong (S'99-M'01-SM'08) received the BEng, the MPhil, and the PhD degrees, all in Electrical and Electronic Engineering, from the Hong Kong University of Science and Technology, Hong Kong, in 1996, 1998, and 2001, respectively. After graduation, he joined the Department of Electrical and Electronic Engineering, the University of Hong Kong as a Research Assistant Professor. From July 2003 to December 2003, he visited the Wireless Communications Research Department of Lucent Technologies, Bell-Labs, Holmdel, NJ, U.S. where he was a Visiting Research Scholar studying optimization in broadcast MIMO channels. After that, he then joined the Smart Antennas Research Group of Stanford University as a Visiting Assistant Professor conducting research on overloaded MIMO signal processing. From 2005 to August 2006, he was with the Department of Engineering, the University of Hull, U.K., as a Communications Lecturer. Since August 2006, he has been with University College London Adastral Park Campus where he is a Senior Lecturer.

Dr Wong won the IEEE Vehicular Technology Society Japan Chapter Award of the International IEEE Vehicular Technology Conference-Spring in 2000, and was also a co-recipient of the First Prize Paper Award in the IEEE Signal Processing Society Postgraduate Forum Hong Kong Chapter in 2004. In 2002 and 2003, he received, respectively, the SY King Fellowships and the WS Leung Fellowships from the University of Hong Kong. Also, he was awarded the Competitive Earmarked Research Grant Merit and Incentive Awards in 2003-2004.

Dr Wong is a senior member of IEEE and is also on the editorial board of IEEE TRANSACTIONS ON WIRELESS COMMUNICATIONS. He has worked in several areas including multiuser mobile networks, information theory, smart antennas, space-time processing/coding and channel equalisation. His current research interests center around secure wireless communications theory, cross-layer optimisation, performance analysis of MIMO channels, cooperative wireless networks, cognitive radio, multiuser communications theory, information theory and optimisation. 


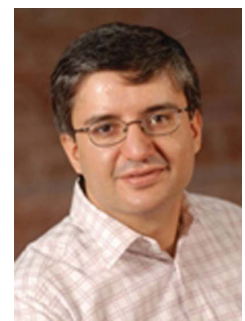

Mohamed-Slim Alouini (S'94, M'98, SM'03, FÕ09) was born in Tunis, Tunisia. He received the $\mathrm{Ph} . \mathrm{D}$. degree in electrical engineering from the California Institute of Technology (Caltech), Pasadena, CA, USA, in 1998. He was with the department of Electrical and Computer Engineering of the University of Minnesota, Minneapolis, MN, USA, then with the Electrical and Computer Engineering Program at the Texas A\&M University at Qatar, Education City, Doha, Qatar. Since June 2009, he has been a Professor of Electrical Engineering in the Division of Physical Sciences and Engineering at KAUST, Saudi Arabia, where his current research interests include the design and performance analysis of wireless communication systems.

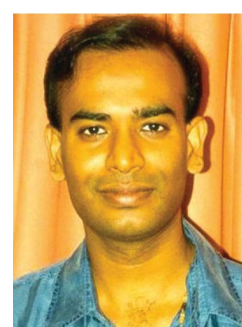

T. Ratnarajah (S'94-A'96-M'05-SM'05) T. Ratnarajah holds B. Eng. (Hons), M.Sc. and Ph.D. degrees. He is currently a Principal Research Engineer with the Institute for Electronics, Communications and Information Technologies (ECIT), Queen's University Belfast, Belfast, UK. Since 1993, he has held various research positions with University of Ottawa, Ottawa, ON, Canada, Nortel Networks, Ottawa, ON, Canada, McMaster University, Hamilton, ON, Canada and Imperial College, London, UK. His research interests include random matrices theory, information theoretic aspects of MIMO channels and ad hoc networks, wireless communications, signal processing for communication, statistical and array signal processing, biomedical signal processing and quantum information theory. He has published over 90 publications in these areas and holds four US. patents. Dr Ratnarajah is a member of American Mathematical Society and Information Theory Society. 